\title{
ZU DEN GRUNDLAGEN DER KOGNITIVEN SPRACHDIDAKTIK IM AKTUELLEN GRAMMATIK-STUDIO
}

Prof. Dr. Jörg ROCHE

Institut für Deutsch als Fremdsprache

Ludwig-Maximilians-Universität München

München, Deutschland, roche@daf.Imu.de

\section{ЗАМОНАВИЙ ГРАММАТИКАДА КОГНИТИВ ЛИНГВОДИДАКТИКА АСОСЛАРИ Йорг РОШ \\ Чет тили сифатида немис тили профессори \\ Людвиг Максимилиан номидаги Мюнхен университети \\ Мюнхен, Германия}

\section{THE BASICS OF COGNITIVE LINGUO-DIDACTICS IN THE CURRENT GRAMMAR STUDIES}

\section{Prof. Dr. Jörg ROCHE}

Institute for German as a Foreign Language

Ludwig-Maximilians-University of Munich

Munich, Germany roche@ daf.Imu.de

UDC (УЎК, УДК): 37.02:81'232

\section{For citation (иқтибос келтириш} учун, для цитирования):

Dr. Jörg Roche. Zu den Grundlagen der Kognitiven Sprachdidaktik im Aktuellen Grammatik-Studio // Foreign Languages in Uzbekistan, 2021, No. 2 (37), pp. 5-11.

\section{https://doi.org/10.36078/1620220502}

Received: January 12, 2021

Accepted: April 17, 2021

Published: April 20, 2021

Copyright (C) 2021 by author(s) and Scientific Research Publishing Inc. This work is licensed under the Creative Commons Attribution International License (CC BY 4.0).

http://creativecommons.org/licenses/by $\not 4.0 /$

\section{Open Access}

\section{Grammatik, Konzeptualisierung und Metapher}

In den Köpfen von Lernern und Lehrkräften spielt die Grammatik eine der wichtigsten Rollen beim Erwerb einer fremden Sprache. Aber wie macht man Schülern transparent, was Grammatik symbolisieren soll? Aus unterschiedlichen Grammatikansätzen (zum Beispiel Textgrammatik, Valenzgrammatik, funktionale Grammatik, generative Grammatik) wissen wir, dass sich Sprache ganz unterschiedlich fassen und ihre Systematik sich dementsprechend unterschiedlich beschreiben lässt. Wir wissen auch, dass einige Ansätze für die Sprachvermittlung besser tauglich sind als andere. Aus Lernersicht sind die abstrakten, oft schulgrammatischen (auf der lateinischen Grammatik basierenden) Systembeschreibungen jedoch meist wenig hilfreich, weil die Beschreibungen meist wenig intuitiv, praktikabel und funktional sind. Man denke nur an die stereotype Vorstellung von der so ,schweren deutschen Sprache" und den oft frustrierend langen Grammatikunterricht. Oft sind die Regeln gar nicht mal (vollständig) richtig, sondern werden ad hoc ,erfunden“. Neuere kognitionslinguistische Ansätze für die Grammatikvermittlung bieten hier eine Lösung, denn sie beruhen auf der Annahme, dass alle grammatischen Strukturen eine Bedeutung haben. Damit soll eine hohe Nachvollziehbarkeit der verwendeten Symbole und Regeln erreicht werden. 
Grammatik kann damit als ein konzeptuell motiviertes System symbolischer Strukturen verstanden werden, das allgemeine Wahrnehmungsprinzipien widerspiegelt und sich nach konzeptuellen Archetypen aus körperlichen Erfahrungen organisiert (z.B. Bewegung, Kraft, Raum etc.), die jedoch unterschiedlich gewichtet werden (Langacker 2008a, b). Meteorologische Erscheinungen wie Schnee, Regen oder die Hitze der Sonne werden so zum Beispiel im Deutschen vorwiegend als Behälter konzeptualisiert (im Regen, in der Sonne etc.), während sie in romanischen Sprachen als Fläche/Dach ausgedrückt werden (bajo el sol, bajo la lluvia, sous le soleil, sous la pluie). Sprachen wie das Russische kombinieren sogar mehrere körperliche Erfahrungen zur Beschreibung desselben Phänomens: Neben der Konzeptualisierung der Sonne als Behälter können sich Sprecher des Russischen auch dafür entscheiden, die besonnte Oberfläche auf dem Boden zu profilieren (vgl. hierzu auch die Einführung in die Spracherwerbsforschung und die Fremdsprachendidaktik in Roche 2020).

Solche körperlichen Erfahrungen und mentalen Bilder werden zwar in den verschiedenen Sprachen unterschiedlich verwendet, allen Sprachen ist jedoch der dahinter liegende Prozess der Metaphorisierung gemeinsam, nach dem ein bestimmter konzeptueller Inhalt von einer Quellendomäne auf eine Zieldomäne übertragen wird (Lakoff \& Johnson 1980; Roche \& Roussy-Parent 2006). Oft basieren die Quellendomänen auf konkreten Konzepten, wie zum Beispiel Druck, Kraft, Vertikalität etc., und die Zieldomänen auf abstrakten Konzepten, wie zum Beispiel der Teilnahme an einer Veranstaltung. Metaphern sind dynamisch und produktiv und können sich in allerlei Kontexten als ein wichtiges Mittel zum Ausdruck komplexer abstrakter Sachverhalte erweisen. Da aber beim Verstehen solcher metaphorischen Konstrukte auch der soziokulturelle und der pragmatische Kontext eine wichtige Rolle spielen, kann ihre erfolgreiche Erschließung nicht alleine durch konzeptuelle Prozesse sichergestellt werden (de Cock \& Suñer Muñoz 2018; Kövecses 2015: 15). Vielmehr situieren sich unbekannte Metaphern auf einem Kontinuum, das einerseits aus universellen körperlichen Erfahrungen und andererseits aus kontextbedingter, kulturspezifischer Variation besteht (Kövecses 2015: 14; vergleiche auch Kövecses 2010). Für eine erfolgreiche Erschließung sollte also der konzeptuelle Inhalt der Metapher mit beiden Polen, der Universalität und der Kontextspezifik, vereinbar sein. Aus diesem Grund ist auch die sogenannte Landeskunde im Fremdsprachenunterricht so fundamental wichtig.

Da die Konzeptualisierung letztlich den Prozess der Formulierung von sprachlichen Nachrichten steuert, kann auch nur die angemessene Konzeptualisierung den Ausgangspunkt für die Grammatikvermittlung darstellen (vgl. Roche \& Suñer Muñoz 2014). Es geht also um die Vermittlung oder den Erwerb einer Kulturgeprägten konzeptuellen Kompetenz (conceptual fluency, metaphoric competence, Danesi 1995).

\section{Grammatik-Animationen}


Bei der Konzeptualisierung spielen Raumkonzepte eine wesentliche Rolle, weil Raum im richtigen Leben und damit in der Sprache implizit oder explizit omnipräsent ist, weil jeder Mensch Raum in seinem Leben erfährt (Mehrdimensionalität, Schwerkraft), weil sich räumliche Metaphern (aus diesem Grund) in vielen Bereichen der Sprache finden (zum Beispiel in temporalen Angaben oder in lexikalischen Elementen wie ,hochladen', ,herunterkommen', die oberen $10.000^{\circ}$ ), weil sich räumliche Verhältnisse anschaulich darstellen und durch physische Handlungen begleiten lassen (Szenarien, Theaterpädagogik). In Grammatikanimationen mit Sportmetaphern lassen sich derartige Grammatikkonzepte nachweislich signifikant besser vermitteln als mit traditionellen Methoden (Compaoré 2018; EL-Bouz 2016; Gradel 2016, 2017, 2020; Kanaplianik (EL-Bouz) 2016; Roche \& EL-Bouz 2018b; Roche 2013; Scheller 2009; Suñer Muñoz 2013; Hoffmann 2018, Suñer Muñoz \& Roche 2019; siehe auch das Aktuelle Grammatikstudio unter www.granima.de). Die Grundlagen der kognitiven Sprachdidaktik sind inzwischen ebenfalls gut und ausführlich dokumentiert und erläutert (Roche \& Suñer Muñoz 2014, 2017, 2019). Hier ein bekanntes Beispiel aus dem Aktuellen Grammatikstudio (Roche \& EL-Bouz 2018a, b):

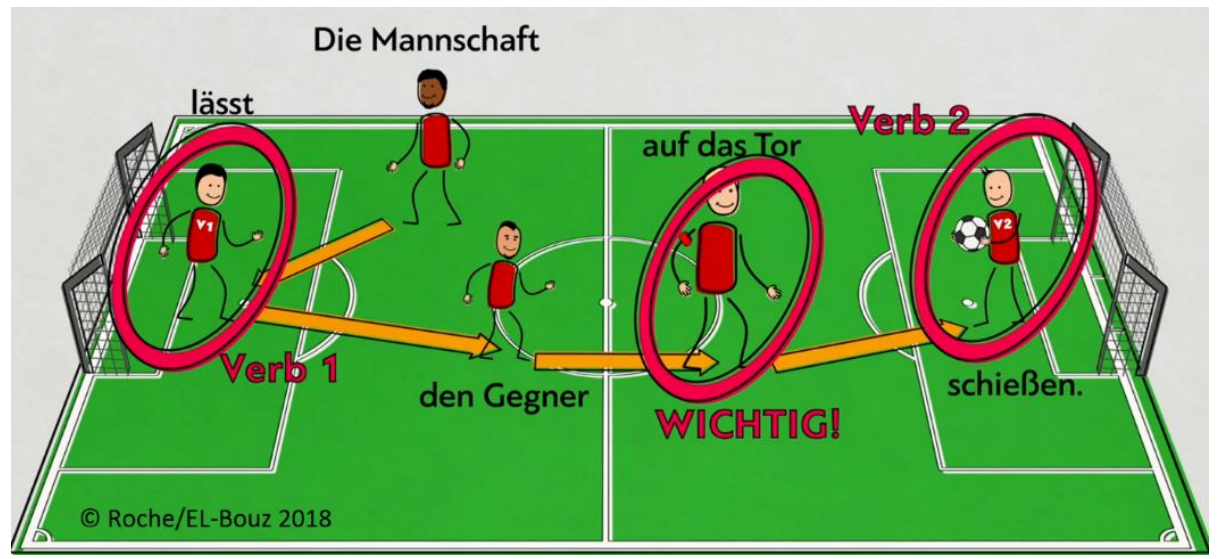

Abb.1: Deutsche Wortfolge (Satzklammer) am Beispiel des Fußballspiels: Phase 1 der Erklärungen: Satzrahmen durch Torwarte, Größe der Spieler symbolisiert Betonung/Salienz (granima.de)

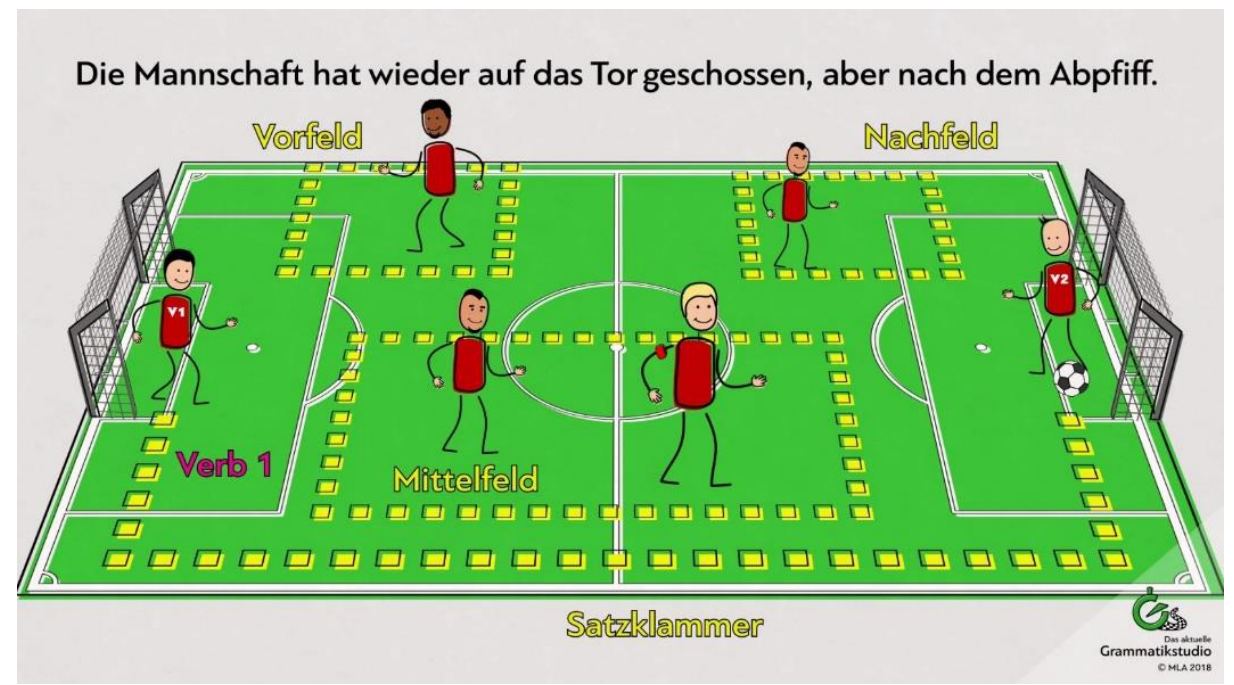


Abb. 2: Deutsche Wortfolge (Satzklammer) am Beispiel des Fußballspiels: Phase 2 der Erklärungen mit Metasprache (granima.de)

Auch alle anderen Phänomene der deutschen Grammatik lassen sich mit Sportanimationen darstellen und erklären. So kann die Nutzung von Akkusativ und Dativ bei den Wechselpräpositionen mit Hilfe einer Grenzüberschreitung visualisiert werden: wird eine Grenze überschritten, verwendet das Deutsche den Akkusativ (ich gehe in die Schule), wird die Grenze nicht überschritten - mit oder ohne Bewegung -, verwendet man den Dativ (ich renne/fahre in der Schule, z.B. auf dem Schulhof). Auch die Hilfsverben haben und sein lassen sich mit der Grenzüberschreitung erklären. Bewegungsverben wie fahren, schwimmen, laufen, fliegen ... können daher mit beiden Hilfsverben realisiert sein. Der Unterschied liegt darin, ob eine Überschreitung einer Grenze impliziert oder expliziert ist. Hier zwei Darstellungen aus dem Hobbyleben eines DAF-Dozenten:

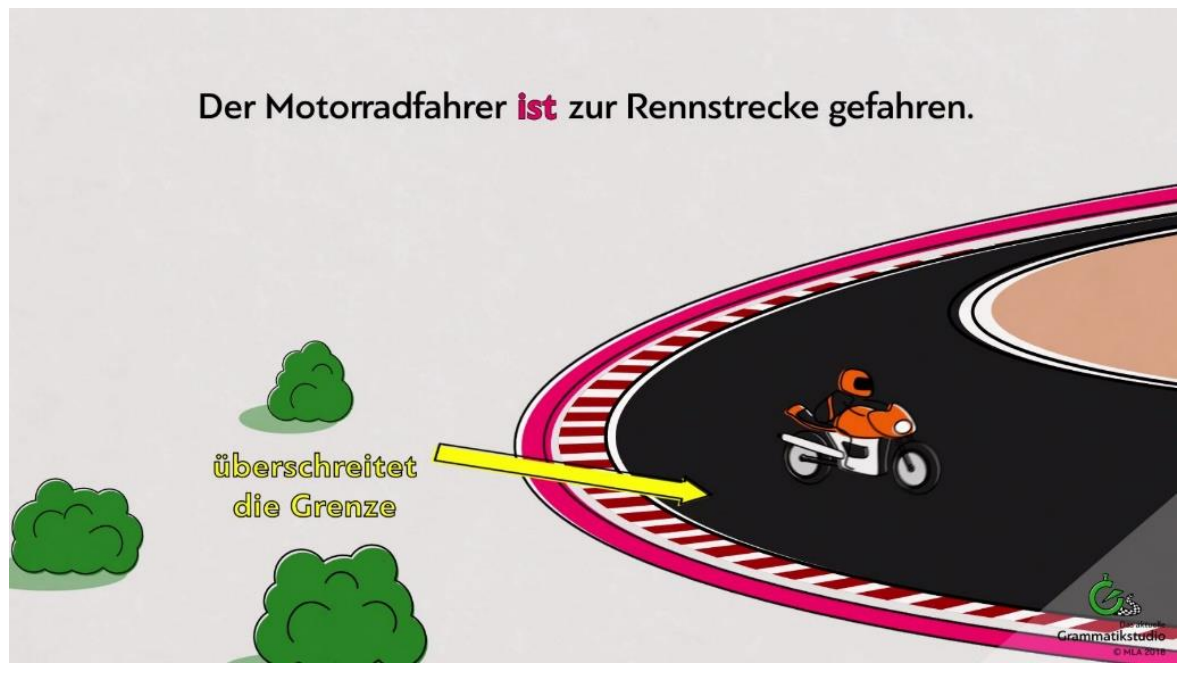

Abb. 3: Perfekt mit sein: Erklärung anhand einer Grenzüberschreitung (granima.de)

Der Motorradfahrer hat 30 Runden gefahren.

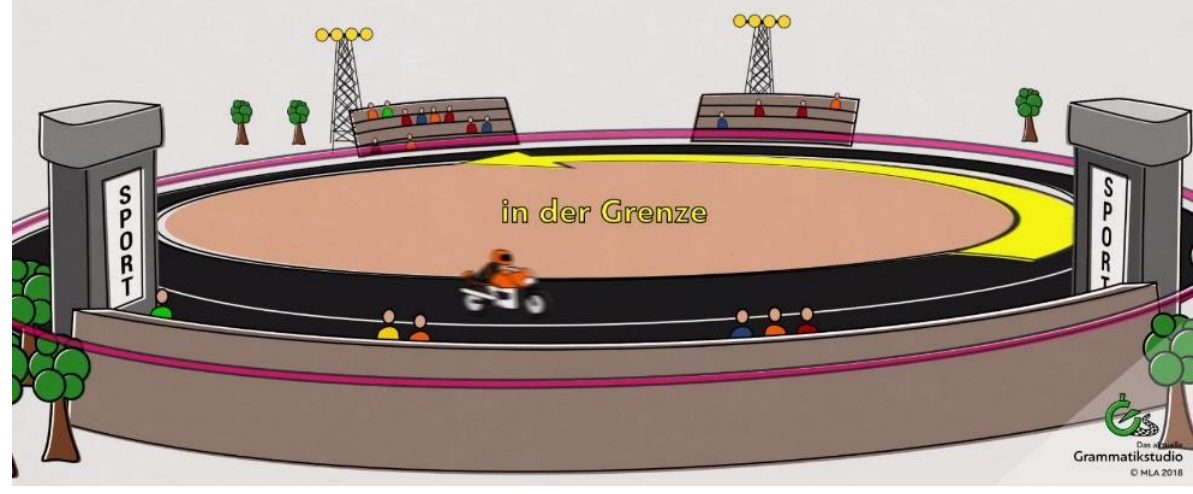

Abb. 4: Perfekt mit haben: Erklärung anhand einer Grenze (granima.de) 


\section{Unterrichtsvorschläge und Ausblick}

Neben traditionellen Übungen bieten Grammatikanimationen dank ihrer physischen Darstellbarkeit und ihrem Bezug auf konkrete Situationen mehrere einfache Optionen an, sowohl für die Erklärung als auch für die Einübung des Materials:

- Die Lerner können neue Sätze oder Situationen mit den grammatischen Merkmalen nennen und sie zeichnen, an der Tafel oder im Heft. Dabei sollten konzeptuelle Elemente, die für die Wahl entscheidend sind, hervorgehoben werden (Grenzen des Hintergrunds, Bewegung der Figur, Druck-Gegendruck etc.).

-Viele Situationen z.B. mit räumlichen Präpositionen können im Klassenraum mit den vorhandenen Gegenständen auch nachgespielt werden. Dabei sollten die Eigenschaften der Figuren und Hintergründe, die Kraftübertragung beim Passiv oder die Intensität des Druckes bei Modalverben ebenso betont werden (der Tisch ist eine horizontale Oberfläche, der gesellschaftliche Druck in sie muss arbeiten ...).

- Sollte eine Kombination von Deutsch- und Sportunterricht oder einer anderen Form inhaltsbasierten Lernens möglich sein, bietet die Sporthalle, der Schulhof, die Werkstatt etc. einen perfekten Ort an, um räumliche Präpositionen und andere Grammatik $\mathrm{zu}$ üben. Beispielsweise können die Lerner mit dem Sportinventar spielen und Übungen ausführen und dabei laut nennen, was sie machen (Ich schieße den Ball ins Tor / neben das Tor; Ich laufe die Linie entlang). Hier ist der Kreativität und dem sportlichen (und linguistischen) Geist der Lerner und auch Lehrkräfte viel Platz geboten.

- Außerdem können Lerner die Granima App mit selbstkorrigierenden Übungen im Google Play Store und im App Store von Apple kostenfrei herunterladen. (Die Animationen sind in einzelnen Paketen für einen geringen Beitrag verfügbar über die Webseite der Multilingua-Akademie und den Webshop des hueberVerlages).

An anderer Stelle wurde gezeigt (Roche \& EL-Bouz 2018b), wie Animationen auch stärker in handlungsorientierte Lernkontexte eingebettet sein können (siehe auch Niemeier 2017 sowie die Embodiment-Studie von Suñer Muñoz \& Roche (2019)). Die hier skizzierte Darstellungsweise eignet sich also für das ganze Spektrum einer induktiven und deduktiven Präsentation und nicht nur für die polaren Endpunkte explizit - implizit. Sie lässt sich sehr gut ebenfalls mit einer handlungsorientierten Sprachdidaktik verbinden, um sprachliche und konzeptuelle Strukturen zu vermitteln, bewusst zu machen, zu erproben und im richtigen Leben korrekt zu verwenden.

\section{Literatur}

1. de Cock, Barbara \& Suñer Muñoz, Ferran (2018): The influence of conceptual differences on processing taboo metaphors in the foreign language. In: Pizarro Pedraza, Andrea (Hrsg.): Linguistic Taboo Revisited. Novel Insights from Cognitive Perspectives. Cognitive Linguistics Research. Volume 61. Berlin: de Gruyter, 201-222.

2. Compaoré, Clément (2018): Evaluation von eLernprozessen. Eine empirische Untersuchung am Beispiel des Einsatzes kognitionsbasierter Grammatikanimationen zum kollaborativen Lernen der deutschen Grammatik in virtuellen Klassen. Berlin, Münster: LIT. 
3. Danesi, Marcel (1995): Learning and teaching languages: the role of "conceptual fluency". International Journal of Applied Linguistics 5: 1, 3-20.

4. EL-Bouz, Katsiaryna (2016): Animation of grammar. Interplay of cognitive linguistics and multimedia learning: the example of German modal auxiliaries. In: Goschler, Juliana \& Niemeier, Susanne (Hrsg.): Yearbook of the German Cognitive Linguistics Association. Berlin, Boston: de Gruyter, 135-151.

5. Gradel, Valentina (2016): The acquisition of the German case system by foreign language learners through computer animations based on cognitive linguistic. In: Goschler, Juliana \& Niemeier, Susanne (Hrsg.): Yearbook of the German Cognitive Linguistics Association. Berlin, Boston: de Gruyter, 113-134.

6. Gradel, Valentina (2017): 72 Adjektivendungen zum Auswendiglernen. In: Zhu, Jianhua; Zhao, Jin \& Szurawitzki, Michael (Hrsg.): Akten des XIII. Internationalen Germanistenkongresses Shanghai 2015 - Germanistik zwischen Tradition und Innovation. Frankfurt am Main, Bern, Bruxelles, New York, Oxford, Warszawa, Wien: Peter Lang, 21-30.

7. Gradel, Valentina (2020): Der Erwerb der Adjektivdeklination durch kognitionslinguistisch basierte Apps im Unterricht des Deutschen als Fremdsprache. Münster: LIT.

8. Hoffmann, Isabel (2018): Räumlich konzeptualisierte Funktionsverbgefüge - eine Erwerbsstudie. Zeitschrift für Interkulturellen Fremdsprachenunterricht 23: 2, 74-85. https://tujournals.ulb.tu-

darmstadt.de/index.php/zif/article/view/921/922 (09.02.2021).

9. Kanaplianik (EL-Bouz), Katsiaryna (2016): Kognitionslinguistisch basierte Animationen für die deutschen Modalverben. Zusammenspiel der kognitiven Linguistik und des multimedialen Lernens bei der Sprachvermittlung. Berlin, Münster: LIT.

10. Kövecses, Zoltán (2010): Metaphor: A Practical Introduction. Oxford: Oxford University Press.

11. Kövecses, Zoltán (2015): Where Metaphors Come from. Reconsidering Context in Metaphor. Oxford: Oxford University Press.

12. Lakoff, George \& Johnson, Mark (1980): Metaphors We Live by. Chicago: University of Chicago Press.

13. Langacker, Ronald W. (2008a): Cognitive Grammar. A Basic Introduction. Oxford: Oxford University Press.

14. Langacker, Ronald W. (2008b): Cognitive grammar as a basis for language instruction. In: Robinson, Peter \& Ellis, Nick (Hrsg.): Handbook of Cognitive Linguistics and Second Language Acquisition. New York: Routledge, 66-88.

15. Niemeier, Susanne (2017): Task-based Grammar Teaching of English. Where Cognitive Grammar and Task-based Language Teaching Meet. Tübingen: Narr.

16. Roche, Jörg (2013): Mehrsprachigkeitstheorie. Erwerb - Kognition - Transkulturation - Ökologie. Tübingen: Narr.

17. Roche, Jörg (2020): Fremdsprachenerwerb und Fremdsprachendidaktik. 4. völlig neu bearbeitete Auflage. Tübingen: UTB Basics.

18. Roche, Jörg \& EL-Bouz, Katsiaryna (2018a): Das aktuelle Grammatikstudio. Mit Konzeptvorlagen von Ferran Suñer Muñoz (Passiv, Funktionsverbgefüge), Valentina Gradel (Adjektiv) und Isabel 
Hoffmann (Funktionsverbgefüge). Hrsg. von Roche, Jörg. München: Multilingua Akademie. www.granima.de.

19. Roche, Jörg \& EL-Bouz, Katsiaryna [unter Mitarbeit von Oliver Leuchte] (2018b): Deutsche Grammatik sportlich und animiert. Zeitschrift für Interkulturellen Fremdsprachenunterricht 23: 1, 30-42. https://tujournals.ulb.tu-

darmstadt.de/index.php/zif/article/view/877/878 (09.02.2021).

20. Roche, Jörg \& Roussy-Parent, Melody (2006): Zur Rolle der kontrastiven Semantik in interkultureller Kommunikation. Fremdsprachen Lehren und Lernen 35, 228-250.

21. Roche, Jörg \& Suñer Muñoz, Ferran (2014): Kognition und Grammatik: Ein kognitionswissenschaftlicher Ansatz zur Grammatikvermittlung am Beispiel der Grammatikanimationen. Zeitschrift für Interkulturellen Fremdsprachenunterricht 19: 2, 119 145.

https://tujournals.ulb.tu-

darmstadt.de/index.php/zif/article/view/36/33 (12.03.2020).

22. Roche, Jörg \& Suñer Muñoz, Ferran (2017): Sprachenlernen und Kognition. Kompendium Deutsch als Fremdsprache / Deutsch als Zweitsprache. Band 1. Tübingen: Narr.

23. Scheller, Julija (2009): Animationen in der Grammatikvermittlung. Multimedialer Spracherwerb am Beispiel von Wechselpräpositionen. Berlin, Münster.

24. Suñer Muñoz, Ferran (2013): Bildhaftigkeit und Metaphorisierung in der Grammatikvermittlung am Beispiel der Passivkonstruktion. Zeitschrift für Interkulturellen Fremdsprachenunterricht 18: 1, 4-20. https://tujournals.ulb.tu-darmstadt.de/index.php/zif/article/view/65/61 (09.01.2021).

25. Suñer Muñoz, Ferran \& Roche, Jörg (2019): Embodiment in concept-based L2 grammar teaching: The case of German light verb constructions. In: v. Jordens, Peter \& Roberts. Leha (Eds.): International Review of Applied Linguistics in Language Teaching. Berlin, Boston: de Gruyter. https://www.degruyter.com/view/j/iral.ahead-of-print/iral-20180362/iral-2018-0362.xml (09.02.2021). 